\title{
The mass function of the Las Campanas loose groups of galaxies
}

\author{
P. Heinämäki ${ }^{1,2}$, J. Einasto ${ }^{1}$, M. Einasto ${ }^{1}$, E. Saar ${ }^{1}$, D. L. Tucker ${ }^{3}$, and V. Müller ${ }^{4}$ \\ 1 Tartu Observatory, 61602 Tõravere, Estonia \\ 2 Tuorla Observatory, Väisäläntie 20, 21500 Piikkiö, Finland \\ ${ }^{3}$ Fermi National Acceleration Laboratory, M8 127, PO Box 500, Batavia, IL 60510, USA \\ 4 Astrophysical Institute Potsdam, An der Sternwarte 16, 14482 Potsdam, Germany
}

Received 18 February 2002 / Accepted 10 October 2002

\begin{abstract}
We have determined the mass function of loose groups of galaxies in the Las Campanas Redshift Survey. Loose groups of galaxies in the LCRS range in mass from $M \sim 10^{12} M_{\odot}$ to $10^{15} M_{\odot}$. We find that the sample is almost complete for masses in the interval $5 \times 10^{13}-8 \times 10^{14} M_{\odot}$. Comparison of the observed mass function with theoretical mass functions obtained from $N$-body simulations shows good agreement with a CDM model with the parameters $\Omega_{\mathrm{m}}=0.3, \Omega_{\Lambda}=0.7$ and the amplitude of perturbations about $\sigma_{8}=0.78-0.87$. For smaller masses the mass function of LCRS loose groups flattens out, differing considerably from the group mass function found by Girardi \& Giuricin (2000) and from mass functions obtained by numerical simulations.
\end{abstract}

Key words. cosmology: observations - cosmology: large-scale structure of Universe

\section{Introduction}

One important constraint of cosmological models is provided by the mass function of clusters and groups of galaxies. In the case of popular $\Lambda \mathrm{CDM}$ models the amplitude and shape of the mass function depend primarily on the mean density of matter in the Universe, $\Omega_{\mathrm{m}}=\Omega_{\mathrm{c}}+\Omega_{\mathrm{b}}$, where $\Omega_{\mathrm{c}}$ and $\Omega_{\mathrm{b}}$ are the mean densities of the cold dark matter and baryonic matter in units of the critical cosmological density, respectively. The amplitude of the mass function also depends on the amplitude of the power spectrum of density fluctuations, which can be characterised by the $\sigma_{8}$ parameter (the linearly extrapolated rms density fluctuations in a sphere of $8 \mathrm{~h}^{-1} \mathrm{Mpc}$ radius). The local abundance of rich clusters of galaxies can be used to estimate $\sigma_{8}$ (Bahcall et al. 1997; de Theije et al. 1998; Cen 1998).

Determination of the cluster mass function consists of two tasks: calculation of cluster masses, and estimation of the spatial density of clusters. Masses of clusters can be derived using either X-ray data and the mass-temperature relation or data from optical surveys using the velocity dispersion of galaxies in clusters (virial masses). In a pioneering study by Bahcall \& Cen (1993; hereafter BC93), both mass determination methods were used. Masses were derived for Abell clusters of all richness classes and for groups of galaxies. Biviano et al. (1993) and Girardi et al. (1998, hereafter G98) calculated virial

Send offprint requests to: $\mathrm{P}$. Heinämäki,

e-mail: pekka@aai.ee masses of nearby clusters. Reiprich \& Böhringer (2002) used a X-ray flux-limited sample of galaxy clusters (HIFLUGCS, the HIghest X-ray FLUx Galaxy Cluster Sample) to obtain a mass function. The spatial density of massive clusters of galaxies, according to G98, exceeds the density found by BC93 by a factor of almost ten. As shown by G98, this difference is mainly due to the fact that BC93 assumed a one-to-one correspondence between the richness of a cluster and its mass, whereas in reality this relationship has a large scatter. A new determination of the mass function of loose groups of galaxies has been provided by Girardi \& Giuricin (2000).

The sample of loose groups of galaxies identified in the Las Campanas Redshift Survey (LCRS) by Tucker et al. (2000, hereafter TUC) provides a possibility to derive a new independent estimate of the mass function of clusters and groups of galaxies. Loose groups represent the most numerous component of galaxy clustering, and so we should be able to determine their statistical properties much better than those for rare rich clusters of galaxies. The virial masses of loose groups of galaxies in the LCRS range from $M \sim 10^{12} M_{\odot}$ to $10^{15} M_{\odot}$; these masses were estimated by TUC using velocity dispersions of galaxies and harmonic radii of groups.

In this paper we shall estimate the mass function of LCRS loose groups. Our main task is to derive the spatial density of groups of various masses. The volume of the LCRS sample is well determined, thus we hope to get an unbiased estimate of the spatial density of groups. We shall compare the empirical 
mass function with theoretical mass functions found using numerical simulations of structure evolution. In our simulations we assign to a halo all the particles identified as members of the halo by halo finder. In observations this means that the velocity dispersion of galaxies is assumed to be identical to that of the dark matter. This comparison allows us to check the consistency of structure evolution models with empirical data and to find the set of cosmological parameters which brings models into agreement with data. We also determine the mass interval where the sample of groups is not influenced by selection effects.

\section{The data}

\subsection{Observations}

The LCRS (Shectman et al. 1996) is an optically selected galaxy redshift survey where a multi-object spectrograph was used to measure simultaneously redshifts of 50 or 112 galaxies. Extending to a redshift of $z \approx 0.2$, the catalogue covers 6 slices of size on average $1.5^{\circ} \times 80^{\circ}$, containing 23967 galaxies with measured redshifts within the official survey photometric and geometric limits. Three slices are located in the northern Galactic hemisphere, centred at declinations $\delta=-3^{\circ}$, $-6^{\circ},-12^{\circ}$, and the other three slices are located in the southern Galactic hemisphere, centred at declinations $\delta=-39^{\circ},-42^{\circ}$, $-45^{\circ}$. TUC applied a friends-of-friends (FoF) percolation algorithm to extract the catalogue of Las Campanas loose groups of galaxies (hereafter LCLG). The linking parameter was chosen to get a density enhancement limit of $\delta n / n \geq 80$. The minimum group membership was chosen to be three.

Because the spectroscopy was carried out for each field either via a 50 or a 112 fibre multiobject spectrograph, the selection criteria varied from field to field. The nominal apparent magnitude limits for 50 fibre fields were $16.0 \leq R \leq 17.3$ and for 112 fibre fields the limits were $15.0 \leq R \leq 17.7$. According to TUC great effort was put forth in accounting for these field-to-field sampling variations. The general properties of the 50 fibre and the 112 fibre groups agree well with properties of groups found from other surveys, thus we shall use the whole TUC group sample.

Only groups with redshifts $10000 \leq c z \leq 45000 \mathrm{~km} \mathrm{~s}^{-1}$ were included into the LCLG sample. TUC concluded that the LCLG is a useful sample for a variety of studies requiring an unbiased collection of loose groups. It is based on the LCRS galaxy sample, which is the first redshift survey that can claim to enclose a reasonably fair sample of the nearby universe.

The complete LCLG list includes 1495 groups. TUC also introduced a "clean sample", where groups with four potential bias factors are excluded: 1) groups which are too close to a slice edge, 2) groups which have the crossing time greater than the Hubble time, 3) groups with the corrected velocity dispersion zero what can happen since TUC subtracted an redshift measurement error of $67 \mathrm{~km} \mathrm{~s}^{-1}$ in quadrature from each group velocity dispersion, and 4) groups containing a 55 arcsec orphan galaxy, i.e. a galaxy with no measured redshift. The last bias was caused by technical reasons (the fibre separation limit, which prevents the observation of neighbouring galaxies within
55 arcsec of each other). In the full sample this effect was compensated for by reintroducing lost galaxies, assigning to them a redshift equal to that of its nearest neighbour, convolved with a Gaussian of width $\sigma=200 \mathrm{~km} \mathrm{~s}^{-1}$.

\subsection{Simulations}

A low density CDM universe with a cosmological constant $(\Lambda \mathrm{CDM})$ is widely regarded as the best model compatible with most of the currently available data; e.g. with the microwave background anisotropy measured by BOOMERANG (de Bernardis et al. 2000) and MAXIMA I (Hanany et al. 2000) experiments, and with data on the large scale structure of the universe: two-point correlation functions and power spectra, and the spatial density of mass-limited samples of galaxy clusters (Governato et al. 1999; Colberg et al. 2000; Pierpaoli et al. 2001).

For the present study we employ a flat cosmological model $\left(\Omega_{\mathrm{m}}+\Omega_{\Lambda}=1\right)$ with the following parameters: the matter density $\Omega_{\mathrm{m}}=0.3$, the baryonic density $\Omega_{\mathrm{b}}=0.04$, the vacuum energy density (cosmological constant) $\Omega_{\Lambda}=0.7$, and the Hubble constant $h=0.7$ (here and throughout this paper $h$ is the present-day Hubble constant in units of $100 \mathrm{~km} \mathrm{~s}^{-1} \mathrm{Mpc}^{-1}$ ). The simulations were performed using a P3M code (Couchman 1991) for a cube of $200 h^{-1} \mathrm{Mpc}$ size and a $256^{3}$ mesh and for the same number of particles; each particle has a mass of $4.0 \times 10^{10} h^{-1} M_{\odot}$. The transfer function was computed using the CMBFAST code by Seljak \& Zaldarriaga (1996). The rms mass density fluctuation parameter of this model is $\sigma_{8}=0.87$.

We also calculated an additional model using the same code with $128^{3}$ particles in a cube of size $L=100 \mathrm{~h}^{-1} \mathrm{Mpc}$, and the cosmological parameters $\Omega_{\mathrm{m}}=0.3, \Omega_{\Lambda}=0.7, \Omega_{\mathrm{b}}=0.05$, and $h=0.65$; this COBE normalised model has the density fluctuation parameter $\sigma_{8}=0.78$.

We used the FoF algorithm to identify CDM halos. The only free parameter in the FoF method is the linking length, which is defined as the maximum separation between particles which are still joined into groups. In our case the linking length was chosen as 0.23 in units of the mean particle separation, which approximately selects the matter density contrast $\delta n / n=80$. This density contrast was the one chosen by TUC to extract the group catalogue from LCRS, and it is typical of the values used in extracting groups from observational galaxy catalogues. While in the Einstein-de Sitter universe the overdensity within the virial radius of a cluster is $\delta n / n=178$, in a low density spatially flat universe with a cosmological constant it is $\delta n / n \approx 178 \Omega_{\mathrm{m}}^{-0.6}$ (White et al. 1993). The corresponding overdensity for our simulation is $\delta n / n \approx 366$. This means that the group sample determined using a low density contrast $(\delta n / n=80)$ contains groups which can be in an uncertain dynamical state and do not have to be virialised. When particles outside the virialised core are included in the groups, the masses of simulated groups may be overestimated. For comparison we also calculated the mass function of publicly available numerical simulations of the Virgo consortium ${ }^{1}$,

${ }^{1}$ http://www.MPA-Garching.MPG.DE/Virgo/ 
where Jenkins et al. (1998) selected FoF-groups with a linking length of 0.2 of the mean inter-particle separation.

\section{Results}

\subsection{Selection effects in the LCLG sample}

At first we discuss selection effects and show how we took them into account when calculating the mass function. There are two main selection effects in the LCRS: 1) observations are performed in a fixed apparent magnitude interval which transforms to a distance dependent absolute magnitude interval, galaxies fainter or brighter than this interval are not included in the survey; 2) depending on the field 50 or 112 galaxies were measured for redshifts, the actual number of galaxies in the magnitude window could be larger, thus the sample is diluted.

The first selection effect makes it impossible for galaxies outside the window to enter the survey. Consequently groups consisting of faint galaxies occur only in the nearest region of the survey; with increasing distance fainter groups gradually disappear from the sample. This effect is seen in Fig. 1 where the total luminosity of LCRS loose groups is shown as a function of distance. This selection effect can be statistically corrected using individual weights in calculation of the group mass function, following the procedure by Moore et al. (1993) and Girardi \& Guiricin (2000). Namely, we weight each group by $w=1 / \Gamma$, where $\Gamma$ is the volume, which corresponds to the absolute magnitude limit of the third brightest galaxy of a group; if $\Gamma>\Gamma_{0}$ (the full volume of a slice), we take $\Gamma=\Gamma_{0}$. The data to calculate these limits have been tabulated by TUC separately for each of the 327 spectroscopic fields of the LCRS. Figure 2 shows the distribution of the normalised weights $w_{0}$ (in units of the full volume of a slice) as a function of group mass. We see that there is a weak relation between cluster mass and the relative weight: massive groups contain as a rule sufficiently bright galaxies and can be observed in the whole volume of the survey, thus for massive clusters the relative weight is closer to unity.

Now we consider the second selection effect. To estimate the degree of dilution of the LCRS we compare the surface density of galaxies of the LCRS with the surface density of the Sloan Digital Sky Survey. Here the goal was to measure all galaxies in the magnitude window. The number of galaxies with measured redshifts in the Early Data Release of SDSS is 70 per square degree, while the LCRS has only 20 galaxies per square degree in the $-6^{\circ}$ slice, and 30-38 per square degree in the rest of slices. The faint end limit in most slices of the LCRS is almost same as for the SDSS (-17.7), thus the difference in number density is due to dilution of the LCRS. Most slices of the LCRS are diluted by a factor of about 2 , and the slice $-6^{\circ}$ by a factor of 3.5. Dilution decreases not only the number of galaxies but also the number of clusters and groups, as the number of galaxies in a group may fall below 3 and the group will not be included in the catalogue. The number of groups in the $-6^{\circ}$ slice is actually lower than in other slices by a factor of about 2 .

However, uniform dilution does not influence strongly the mass function of groups. Firstly, virial masses of groups

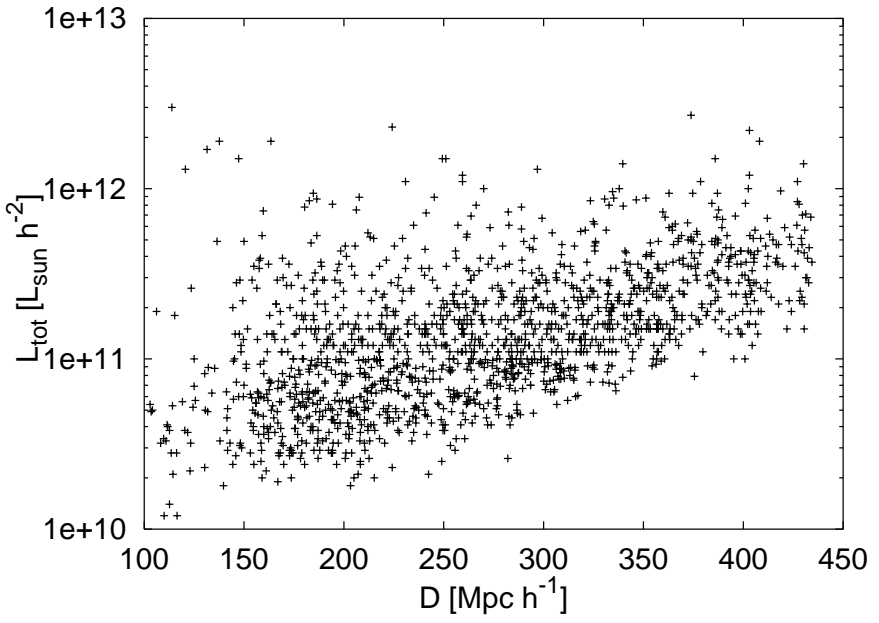

Fig. 1. The total luminosity of the Las Campanas loose groups as a function of their distance.

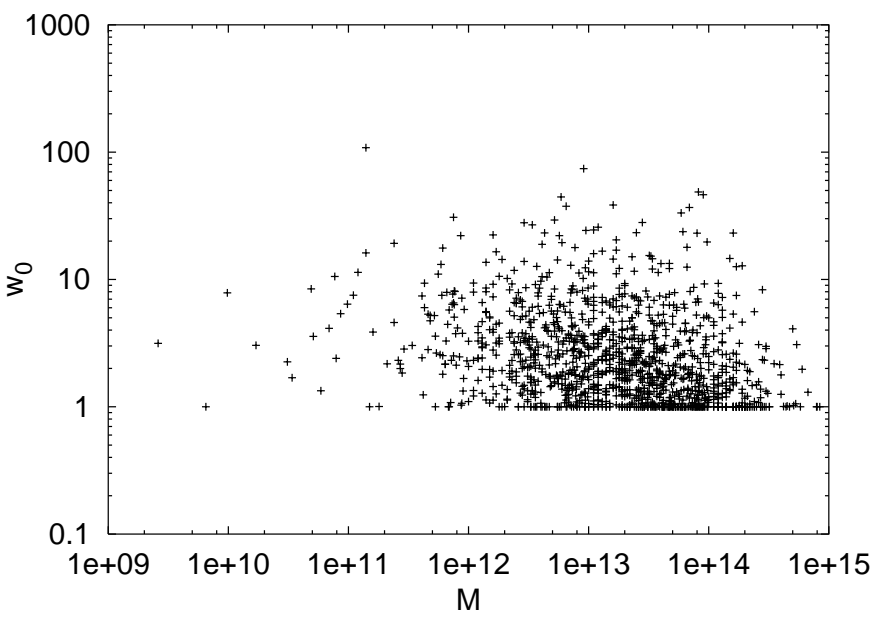

Fig. 2. The weighting parameter $w_{0}$ as a function of the group mass.

practically do not change. When determining virial masses, group members play the role of test particles, which move in a common gravitational potential. Using less test particles gives the same estimate for the potential and for the virial mass as before, only with a larger variance. We checked this conclusion by diluting simulated group catalogues.

Dilution may affect the group catalogue only by reducing the group richness of smaller groups below the catalogue limit. These groups will drop out of the catalogue and the number density of smaller (less massive) groups will fall. We estimated this correction, using group catalogues obtained in our numerical simulations and randomly diluting the groups by $50 \%$, as estimated above. We found that the conditional probability distribution $P(\log N \mid M)$ ( $N$ is the richness of a diluted group, $M$ is the virial mass of the group, and we use decimal logarithms here) is close to a Gaussian with a mean

$\overline{\log N(M)} \approx \log \left(M / M_{\odot}\right)-11.5$

and a rms error $\sigma$ that is also a function of the virial mass,

$\sigma(M) \approx 0.3\left(14-\log \left(M / M_{\odot}\right)\right)$. 
The fraction $\alpha$ of the groups, which remain in the catalogue after dilution, is given then by the error integral:

$\alpha(M)=0.5 \operatorname{erfc}\left(\frac{\log N_{\mathrm{L}}-\overline{\log N(M)}}{\sqrt{2} \sigma(M)}\right)$,

where $N_{\mathrm{L}}$ is the catalogue limit (3 and 5, in our case) and $\overline{\log N(M)}$ and $\sigma(M)$ are given above. In order to restore the original differential mass function, we have to multiply it by the factor $\alpha^{-1}$. This dilution correction is larger than 1 only in the mass interval $10^{12}-10^{13} M_{\odot}$, reaching 1.95 at its lowest end (for $N_{\mathrm{L}}=3$ ) and 2.67 for $N_{\mathrm{L}}=5$. As the differential mass function is small in this mass interval, this correction does not change appreciably the final integral mass function. Also, the dilution correction does not change the high mass end of the mass function at all.

\subsection{Mass function of LCLGs}

The mass function (MF) of galaxy clusters/groups is defined as the number density of clusters above a given mass $M, n(>M)$. To construct the group mass function from a group sample one needs accurate group masses and well defined volume and selection functions of the sample. We have used the masses estimated by TUC, who assumed that the groups were virialised and calculated virial masses of groups as:

$M_{\mathrm{vir}}=\frac{d \sigma_{\mathrm{los}}^{2} R_{\mathrm{h}}}{G}$

where $G$ is the gravitational constant, $R_{\mathrm{h}}$ is the harmonic radius of the group, $\sigma_{\text {los }}$ is the group line-of-sight velocity dispersion, and $d=6$ in the case of a spherically symmetric velocity distribution of the group.

We calculated the volumes of slices as follows:

$V=\frac{\cos \delta_{\mathrm{m}} \Delta \alpha \Delta \delta}{3}\left(r_{2}^{3}-r_{1}^{3}\right)$

here $\delta_{\mathrm{m}}$ is the mean declination of the slice, $\Delta \delta$ and $\Delta \alpha$ are the widths of the slice in declination and right ascension (in radians), and $r_{1}$ and $r_{2}$ are the lower and upper distance used in calculations. This formula is valid for spatially flat $(k=0)$ cosmologies.

The comoving cosmological distance $r$ is a function of measured redshift and depends on the cosmological model as (e.g. Peebles 1993):

$r=\frac{c}{H_{0}} \int_{0}^{z} \frac{\mathrm{d} z^{\prime}}{E\left(z^{\prime}\right)}$

where the function $E(z)$ is given by

$E^{2}(z)=\Omega_{\mathrm{m}}(1+z)^{3}+\left(1-\Omega_{\mathrm{m}}-\Omega_{\Lambda}\right)(1+z)^{2}+\Omega_{\Lambda}$.

The LCLG survey extends up to the redshift $z \sim 0.15$. Tucker et al. (2000) used the Einstein-de Sitter cosmology (assuming $\Omega_{\mathrm{m}}=1, \Omega_{\Lambda}=0$ ) to determine distances in the LCLG survey, while modern data prefer the parameters $\Omega_{\mathrm{m}}=0.3$ and $\Omega_{\Lambda}=0.7$. Thus for the present study we recalculated comoving distances for all galaxies of the LCLG survey. For the particular redshift range $10000 \leq c z \leq 45000 \mathrm{~km} \mathrm{~s}^{-1}$ the ratio between the comoving volumes in these two models is about 0.8 .
Table 1. The numbers of groups in the LCLG catalogue. Columns give the mean declination of a slice, the number of all groups by TUC, the number of groups in the clean sample and the volume of the slice.

\begin{tabular}{lccc}
\hline \hline Slice $\delta$ & TUC & Clean & Volume $\left[\mathrm{Mpc}^{3} / h^{3}\right]$ \\
\hline$-3^{\circ}$ & 288 & 80 & 927902 \\
$-6^{\circ}$ & 147 & 37 & 823452 \\
$-12^{\circ}$ & 276 & 73 & 874202 \\
$-39^{\circ}$ & 249 & 71 & 971817 \\
$-42^{\circ}$ & 257 & 69 & 988435 \\
$-45^{\circ}$ & 256 & 63 & 941531 \\
Total & 1473 & 393 & 5527339 \\
Mean & & & 921223 \\
\hline
\end{tabular}

The numbers of groups for all slices, for the clean sample and the volume of each slice in the LCLG catalogue are shown in Table 1. Only groups with defined mass are included (for some groups the velocity dispersion, corrected for random errors, is zero and mass determination is impossible).

The MF was constructed by sorting the masses in logarithmic bins of width 0.2. Errors in group masses (an estimate of the rms error in virial mass has been found by TUC) were accounted for by convolving group masses with a log-normal distribution of the same mode and variance. Figure 3 shows the mass function of LCLG for all six slices. To have a general picture we added slices together and found the average mass function, denoted by a thick solid line. We found that the average mass function and the mass function of the clean sample distributions are very similar, which indicates that the differences between the clean sample and the full sample are unimportant for the MF. We also tried excluding the slice $\delta=-6^{\circ}$ and found that it has only a weak influence on the total MF. In order to better show the differences between various slices we did not correct these mass functions for dilution. The dilutioncorrected final mass function is given in Table 2 and in Fig. 7.

There is a clear difference in the MF between slices. The two northern slices at $\delta=-6^{\circ}$ and $\delta=-12^{\circ}$ differ from others, having the lowest and the highest amplitudes at the faint end of the MF. We suppose that this discrepancy between mass functions are due to differences in dilution, since the slice $\delta=-6^{\circ}$ was observed by the 50 fibre spectrograph only and the $\delta=-12^{\circ}$ slice by the 112 fibre spectrograph only. This discrepancy cannot be removed by a luminosity-weighting procedure. The remaining differences are probably due to cosmic variance. The slices of the LCRS survey intersect large voids and superclusters. In such slices, loose groups form a variety of structures that may influence the observed mass function at its massive end, since the most massive groups (clusters) are associated with supercluster structures.

Figure 3 shows clearly the flattening of mass functions at the low mass end. Such flattening is usually interpreted as the result of incompleteness of a sample (Girardi et al. 1998), although it could also be real, describing a diminishing number of groups of smaller masses. Thus, we have to study the completeness problem in more detail. 


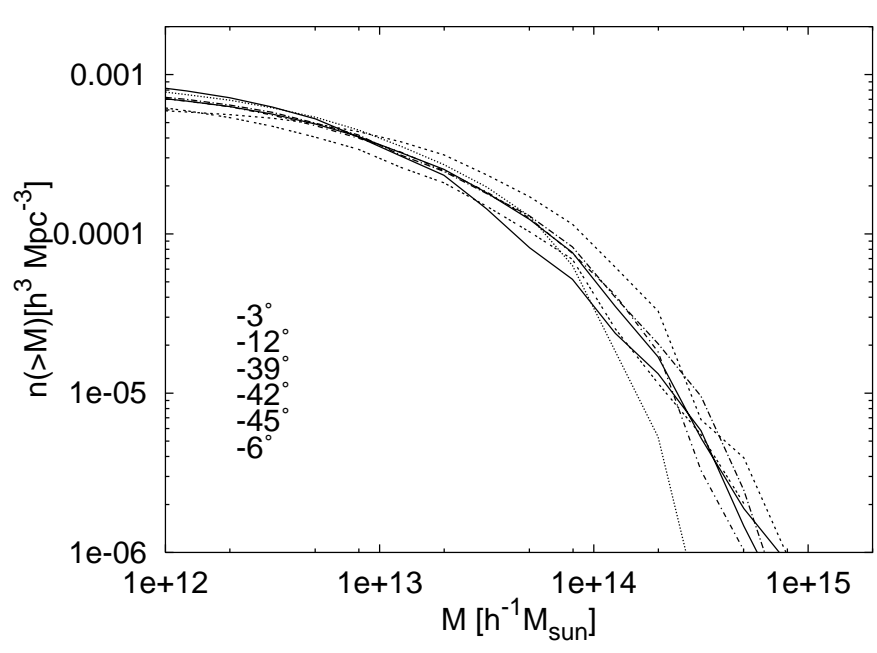

Fig. 3. The cumulative mass functions for all six slices in the LCLG. The thin curves from top to bottom are labelled as seen at the low mass end. The thick solid curve shows the average mass function for all the clusters. These mass functions are not corrected for dilution.

Table 2. The cumulative mass function of LCLG.

\begin{tabular}{lc}
\hline \hline $\log M\left[h^{-1} M_{\odot}\right]$ & Density $\left[h^{3} \mathrm{Mpc}^{-3}\right]$ \\
\hline $1.26 \mathrm{E}+12$ & $7.56 \mathrm{E}-04$ \\
$3.16 \mathrm{E}+12$ & $5.78 \mathrm{E}-04$ \\
$7.94 \mathrm{E}+12$ & $4.09 \mathrm{E}-04$ \\
$1.26 \mathrm{E}+13$ & $3.23 \mathrm{E}-04$ \\
$3.16 \mathrm{E}+13$ & $1.81 \mathrm{E}-04$ \\
$7.94 \mathrm{E}+13$ & $7.63 \mathrm{E}-05$ \\
$1.26 \mathrm{E}+14$ & $3.51 \mathrm{E}-05$ \\
$3.16 \mathrm{E}+14$ & $5.22 \mathrm{E}-06$ \\
$7.94 \mathrm{E}+14$ & $8.77 \mathrm{E}-07$ \\
\hline
\end{tabular}

To estimate the mass completeness limit of the LCLG we can study the volume-density of the groups (within different mass intervals) as a function of distance. In Fig. 4 lines denote mean values of the density in various mass ranges. Any rapid fall of the lines indicates incompleteness for that mass interval. We find from the Fig. 4 that below masses of $10^{13.5}-10^{14} M_{\odot}$ our sample is probably incomplete.

Another way to estimate the mass completeness limit of the LCLG is to use the velocity dispersion distribution function, VDF (Fadda et al. 1996). The VDF is defined similarly to the MF, but for the velocity dispersion of groups. Assuming that the VDF can be described by a simple power law function (note that this assumption does not have a clear physical justification), Fadda et al. (1996) set the completeness limit for their sample at the point where the power-law exponent of the VDF starts to change. For the LCLG this point is reached at the line-of-sight velocity dispersion $\sigma_{\text {los }} \approx 250 \mathrm{~km} \mathrm{~s}^{-1}$.

Figure 5 shows the relation between $\sigma_{\text {los }}$ and mass $M$ for the LCLG catalogue. Eq. (1) defines the relation between these quantities via the harmonic radius of the group. The dotted line illustrates a power-law fit:

$\sigma_{\mathrm{los}}=a\left(\frac{h M}{10^{15} M_{\odot}}\right)^{c} \mathrm{~km} \mathrm{~s}^{-1}$

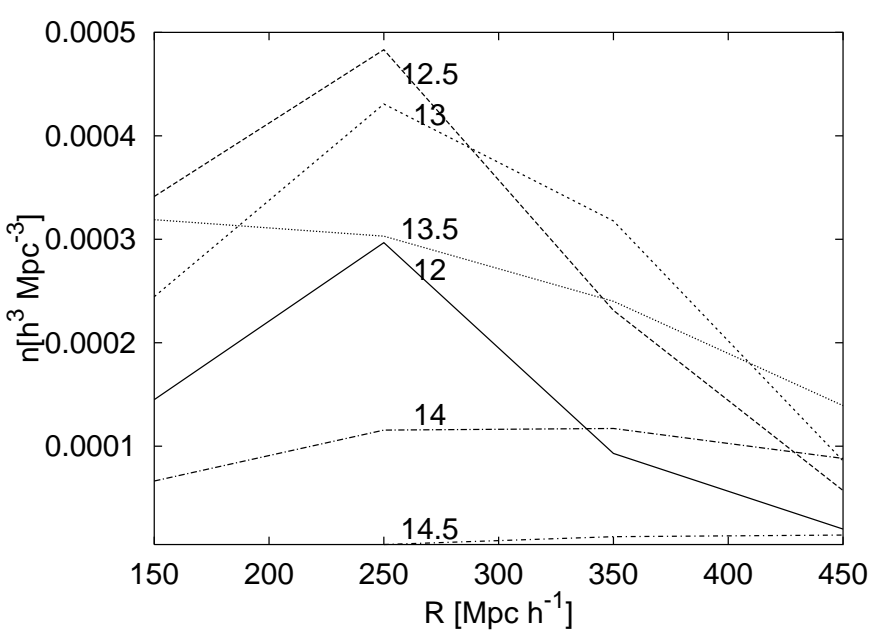

Fig. 4. The volume-density of groups as a function of distance. Each line represents a different mass interval and is labeled by the decimal logarithm of its mean $M / M_{\odot}$.

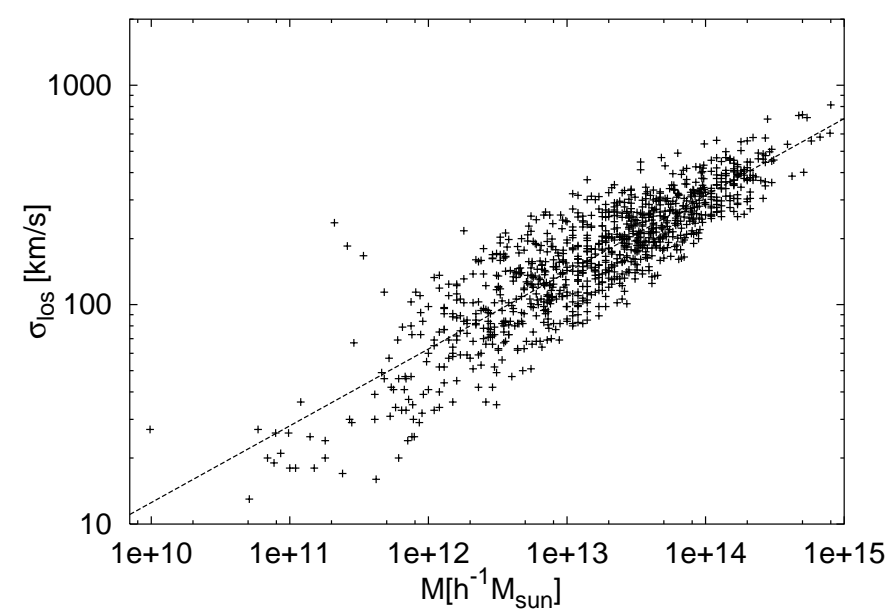

Fig. 5. The velocity dispersion as a function of the mass for the LCLG catalogue. The line shows the analytical (least square) fit.

where $a=819$ and $c=0.35$. The result is in quite good agreement with previous results by del Popolo \& Gambera (2000) and del Popolo et al. (2000), $a=842 \mathrm{~km} \mathrm{~s}^{-1}$ and $c=0.33$, based on $N$-body simulations and X-ray observations. A value $1 / 3$ for the exponent $b$ means that the system is virialised. The fact that the LCLG follow rather closely the virial relation shows that the possible contamination is small and the groups are physical. Setting the completeness limit to $\sigma_{\text {los }}=250 \mathrm{~km} \mathrm{~s}^{-1}$, based on the VDF, in Eq. (5), we get an estimate for the completeness limit in mass between $10^{13} \leq M \leq$ $10^{14} h^{-1} M_{\odot}$, with the mean value about $M \sim 5 \times 10^{13} h^{-1} M_{\odot}$. This result agrees with the result of the first analysis.

\subsection{Reliability of groups}

Ramella et al. (1997) and Girardi \& Giuricin (2000) noted that groups with a few (less than 5 members) may constitute not real, but pseudo-groups. On average, in pseudo-groups the velocity dispersion is considerably larger than in real groups (Ramella et al. 1997). Indeed in the LCLG sample, groups with 
less than 5 galaxies have a very large scatter of velocity dispersions, and some velocity dispersions are extremely large. Analysis of a subsample, where poor groups with only 3 or 4 members are excluded, shows that in the $w-M$ plane (as in Fig. 2) the scatter for this subsample is somewhat smaller than for the whole sample. However, exclusion of all poor groups decreases the mass function considerably in the whole mass range (see Fig. 7, where the mass function is given for two cases, for all groups and for groups with the number of members $\left(N_{\mathrm{m}} \geq 5\right)$. This indicates that at least part of small groups with a large velocity dispersion (and thus a large mass) are probably pseudo-groups (studies by Einasto et al. 2002 indicate that such groups are located mostly in low density regions). On the other hand, most of small groups have velocity dispersions in the same range as richer groups, and are probably real. In other words, exclusion of all small groups introduces an additional selection effect, which is difficult to quantify.

\section{Discussion}

\subsection{Comparison with numerical simulations}

Now we compare our mass function with mass functions found for groups in numerical simulations. Figure 6 shows the velocity dispersion of dark matter particles in groups as a function of the halo (group) mass in our numerical simulations. We see that in small groups with mass less than $\sim 10^{13} h^{-1} M_{\odot}$ (which contain less than $\sim 250$ particles) the scatter of the velocity dispersion is very large. This phenomenon can be explained if we assume that small groups are not yet virialised and are contaminated by non-virialised particles, or alternatively are composed of several small subgroups moving fast relative to each other. Another explanation for this effect is that it is a result of the chosen $\delta n / n-$ the FoF algorithm collects particles from outskirts of small clusters although they do not belong dynamically to the group. The fraction of interlopers in simulated loose groups can become rather high, about $20-30 \%$, as found by Diaferio et al. (1999).

To test the second assumption we built groups, using a lower linking length $b=0.17$ (in units of the mean particle separation). This did not change considerably the scatter of the velocity dispersion among the groups with less than 250 particles. Thus the first explanation is more likely.

To eliminate the effect of unbound groups in simulations we calculated both the potential and kinetic energy for all groups and excluded unbound particles, using the condition $\left|E_{\text {pot }}\right|<0.8 E_{\text {kin }}$ ( $E_{\text {pot }}$ is the potential energy and $E_{\text {kin }}$ the kinetic energy of a group) for an unbound particle. We split groups with a high kinetic energy as compared to the potential energy to separate interacting or merging groups. To this aim we employed a 6D-group finder with a linking measure $\Delta r^{2} / r^{2}+\Delta v^{2} / \sigma_{v}^{2}$, where $r$ and $\sigma_{v}$ are the group effective (halfmass) radius and the velocity dispersion. This procedure affected only about $10 \%$ of the halos, with many of the halos having a small number of DM particles. We show the results of our clean-up procedure in the right panel of Fig. 6, which is similar to the left panel, only non-virialised particles and bypassing groups are excluded. We see that our procedure to eliminate non-virialised particles works well - the low-mass groups follow the same trend as observed for the high-mass groups and clusters.

We have calculated the mass functions for the sample of all groups and for the sample of virialised groups in our simulations. These functions are very similar, only for groups of very small mass $\left(M<10^{12} h^{-1} M_{\odot}\right)$ the MF of virialised groups differs from that of all groups by less than $1 \%$. Thus the contribution of unbound groups to the total mass function is practically negligible.

Figure 7 shows the mass function of dark matter halos in our simulation together with the MF of the LCLG. The Poisson error bars (square root of counts per bin multiplied with the mean weight) for the full LCLG mass function are shown by the dash-dotted lines. In order not to overcrowd the figure, we have not shown the error bars for the $N_{\mathrm{m}} \geq 5$ sample; they are very close to those of the full sample. For small masses $\left(M<5 \times 10^{13} M_{\odot}\right)$ the error bars are smaller than the size of the plotting symbols. For comparison, Fig. 7 shows also the mass function of the simulations by the Virgo Consortium, $\Lambda \mathrm{CDM}$ gif (Jenkins et al. 1998; Kauffmann et al. 1999a). In the Virgo simulation the side of the box was $141.3 \mathrm{~h}^{-1} \mathrm{Mpc}$, the matter density $\Omega_{\mathrm{m}}=0.3$, the cosmological term $\Omega_{\Lambda}=0.7$, the particle mass $1.4 \times 10^{10} M_{\odot} / h$, and the number of the particles $256^{3}$. Figure 7 shows that our $128^{3}$ simulation with the amplitude parameter $\sigma_{8}=0.78$ gives the best fit among the models we used to simulate the LCLG subsample at the massive end $\left(2 \times 10^{14} M_{\odot}-10^{15} M_{\odot}\right)$ of the MF. For smaller masses, down to $4 \times 10^{13} M_{\odot}$ the model with a higher $\sigma_{8}=0.87$ gives a better fit. The cluster MFs in the Virgo Consortium simulation and in our $256^{3}$ simulation are quite similar. For yet smaller masses, where we suspect that the LCLG sample is incomplete, the LCLG mass function flattens out, in contrast to the rising simulated mass functions.

Most of earlier results give $\sigma_{8} \sim 0.9-1$ for the $\Omega_{\mathrm{m}}=$ 0.3 scenario (Eke et al. 1996; Viana \& Liddle 1996, etc.). This value agrees with the recent result by Pierpaoli et al. (2001), $\sigma_{8}=1.02$. Lower values are obtained based on X-ray cluster samples. Reiprich \& Böhringer (2002) derived for $\Omega_{\mathrm{m}}=0.3$, $\sigma_{8} \sim 0.68$. From the ROSAT Deep Cluster Survey Borgani et al. (2001) derived $\Omega_{\mathrm{m}}=0.35$ and $\sigma_{8} \sim 0.66$. Recently Lahav et al. (2002) obtained $\sigma_{8}=0.73$ using the 2dFGRS + CMB data. Finally, Bahcall et al. (2002) used the SDSS data and got $\sigma_{8}=0.72$ for $\Omega_{\mathrm{m}}=0.3$. In principle, $\sigma_{8}$ can be determined on the basis of the CMB data only; future experiments as MAP and PLANCK will help to pinpoint this parameter.

\subsection{Comparison with earlier work}

The mass function determined by Girardi \& Giuricin (2000) is shown in Fig. 7. This group catalogue was constructed using a subsample of galaxies of the Lyon-Meudon Extragalactic Database. Groups were identified using two different methods: the FoF algorithm (P-groups, 453 groups) and the hierarchical method (H-groups, 498 groups). These group samples extend until $c z \leq 5500 \mathrm{~km} \mathrm{~s}^{-1}$. 

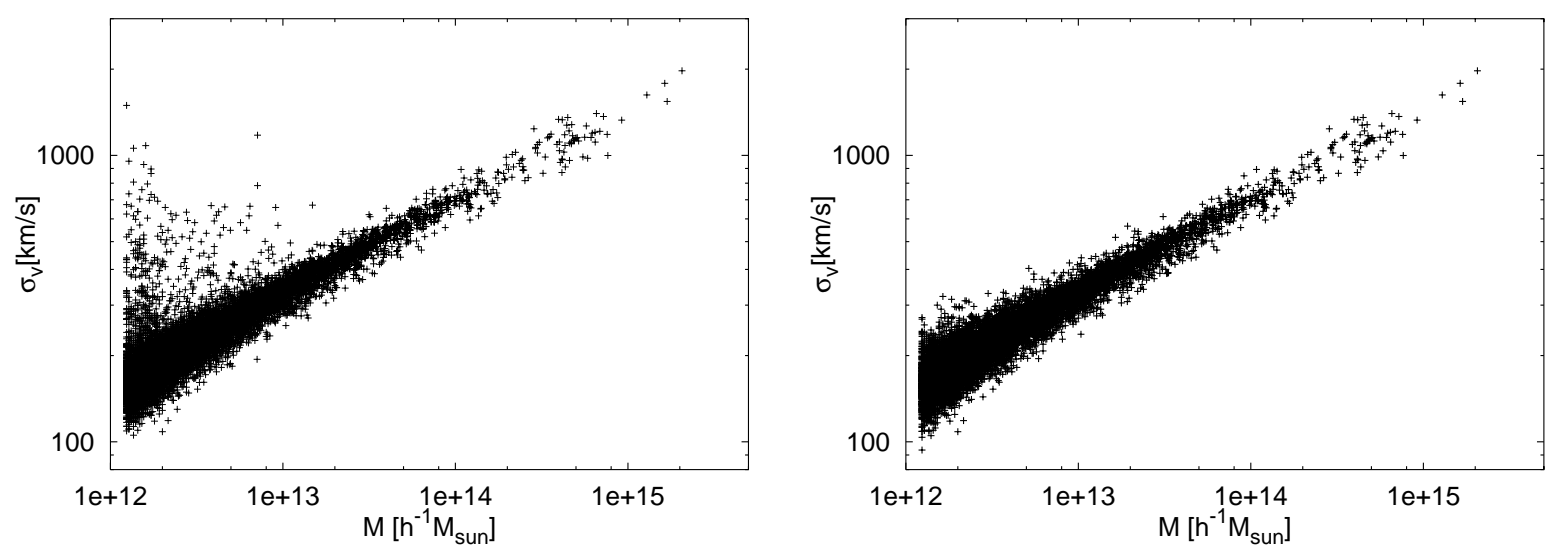

Fig. 6. The left panel shows the velocity dispersion of dark matter particles in halos as a function of the halo mass. The one-to-one correspondence between the number of particles and the halo mass is adopted. The right panel gives the same relation, but unbound groups are removed.

Here we used the P-data from Fig. 3 in Girardi \& Giuricin (2000). In that case they chose the subsample of the nearby groups with $c z \leq 2000 \mathrm{~km} \mathrm{~s}^{-1}$, which they assumed to be a complete sample and a good representation of the total population. However, this sample covers only the local supercluster, while the LCLG catalogue extends up to distances $\sim 450 h^{-1} \mathrm{Mpc}$. This volume contains already a variety of structures.

We see that in the high mass range $\left(M \geq 10^{14} h^{-1} M_{\odot}\right)$ the MFs are rather similar. Around masses $M \approx 5 \times 10^{13}$ the mass functions of the simulations and the LCLG start to differ from each other. Also, at these lower masses the mass function by Girardi \& Giuricin (2000) is considerably higher than the LCLG mass function.

This is an intriguing result that certainly needs further analysis. We see that although loose groups promise to provide better statistics, there are a number of unsolved problems that do not permit a realisation of this promise at the moment. There are two problems in the LCRS group sample: reliable determination of masses of small groups, and selection effects. Virial mass estimates can be in error; Girardi \& Giuricin (2000) try to correct for this. Their correction is rather complicated and could induce its own errors.

The simplest explanation of the difference between the mass functions found in the present paper and that of Girardi \& Giuricin (2000) is a mass-dependent selection effect. The LCRS is a strongly diluted sample of galaxies, and it is biased against low surface brightness galaxies. However, as shown above (Sect. 3.2), dilution practically does not affect the integral mass function.

The second effect, the absence of low-brightness galaxies is probably much less present in the Lyon-Meudon galaxy catalogue, from which Garcia (1998) extracted the catalogue of local groups that served as a source for the Girardi \& Giuricin (2000) mass function. If low surface brightness galaxies were more prevalent in low mass groups - which is plausible - the LCLG mass function would flatten out as seen in Fig. 7.

In a recent paper Martínez et al. (2002) found a similar flattening of the mass function of groups, extracted from the $2 \mathrm{dF}$

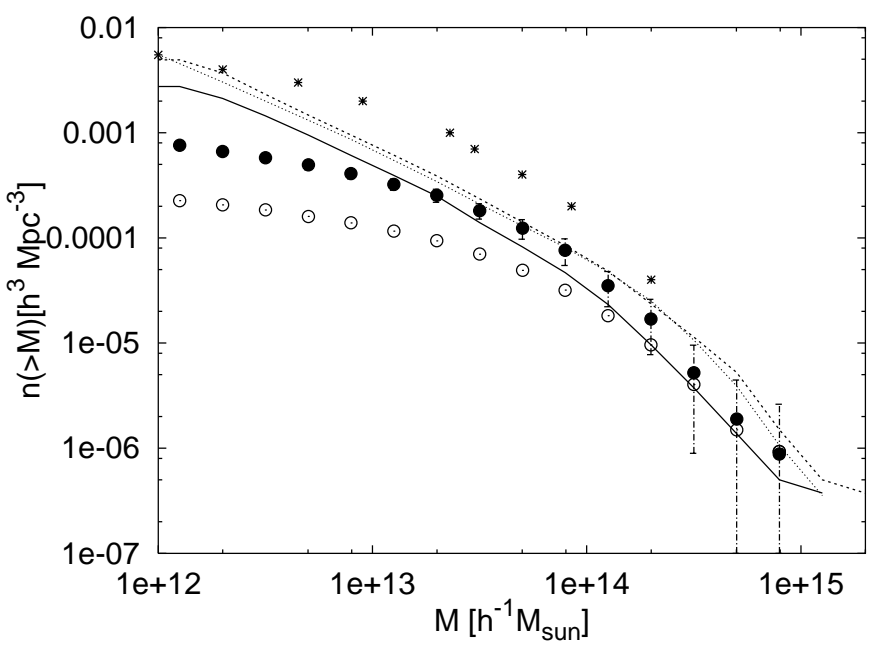

Fig. 7. Comparison of the dilution-corrected observed mass functions and the theoretical mass functions. The observed mass functions are shown with symbols: filled circles (the LCLG whole sample), open circles (the LCLG $N_{\mathrm{m}} \geq 5$ ) and stars Girardi \& Giuricin (2000). The dashed line shows our $256^{3}$ simulation $\mathrm{MF}\left(\sigma_{8}=0.87\right)$, the solid line shows our $128^{3}$ simulation $\left(\sigma_{8}=0.78\right) \mathrm{MF}$, and the dotted line shows the Virgo Consortium simulation, MF. For clarity Poissonian errors are shown only for one case (the full mass function).

catalogue. This is also a deep sample, similar to the LCLG catalogue.

We should also look for possible problems with our simulated mass functions.

One problem is that we are comparing dark matter halos with galaxy-populated halos. It is probable that halos of smaller masses $\left(10^{12}-10^{13}\right)$ can not easily host three or more galaxies. This would explain the deficiency of small-mass galaxy groups. Thus, better simulations of group catalogues will have to include galaxy formation.

There could also be a problem with mass determination. We devoted much attention to accurately evaluate the mass estimates in simulations; in particular, we succeeded in filtering out unbound groups. Still, the mass-velocity dispersion relation of virialised groups widens toward the small mass end, 
showing that virial masses of small groups may have large intrinsic errors. This widening could be caused, first, by the predominance of radial orbits in small groups, but this should be a rather small effect. Another possibility is that small groups might have a higher intrinsic velocity spread than large groups due to different formation histories, or in other words, due to a mixture of young and old groups. This is a question that could be only answered by a detailed dynamical study of observed groups.

Another numerical effect could be an enhanced group production in simulations, caused by gravitational two-body collisions, which were discussed by Suisalu \& Saar (1995) and Splinter et al. (1998). More recent analysis concerning the convergence of dissipationless dark matter $N$-body codes by Knebe et al. (2000) and Power et al. (2002) demonstrates that these effects are small if the gravitational softening and the time steps are chosen carefully.

\section{Conclusion}

In this work we have presented the mass function of the Las Campanas Loose Groups selected by Tucker et al. (2000). We have also studied the mass-velocity dispersion relation that shows the expected scaling relation for virialised systems. We conclude that the loose galaxy groups are basically physical systems. The mass function was compared with results of dark matter simulations.

Our main conclusions are:

1) The completeness interval of the LCLG sample is found to be around $5 \times 10^{13} M_{\odot} / h \leq M \leq 8 \times 10^{14} M_{\odot} / h$.

2) The high end of the mass function of the LCLG sample lies between the simulated mass functions corresponding to $\sigma_{8}=0.78$ and $\sigma_{8}=0.87$.

3) At small masses $M<5 \times 10^{13} M_{\odot} / h$ the mass function of the LCRS sample of loose groups flattens out.

Acknowledgements. The present study was supported by the Estonian Science Foundation grant 4695. P.H. was supported by the Academy of Finland (grant 46733). D.L.T. was supported by the US Department of Energy under contract No. DE-AC02-76CH03000. We thank Mirt Gramann, Sahar Allam and Gert Hütsi for comments. We acknowledge the use of the AP3M code made public by H. Couchman. Finally, we thank our referee for a number of useful and constructive comments.

\section{References}

Bahcall, N. A., \& Cen, R. 1993, ApJ, 407, L49

Bahcall, N. A., Fan, X., \& Cen, R. 1997, ApJ, 485, L53

Bahcall, N. A., et al. 2002 [astro-ph/0205490]

de Bernardis, P., Ade, P. A. R., Bock, J. J., et al. 2000, Nature, 404, 955
Biviano, A., Girardi, M., Giuricin, G., Mardirossian, F., \& Mezzetti, M. 1993, ApJ, 411, L13

Borgani, S., Rosati, P., Tozzi, P., et al. 2001, ApJ, 561, 13

Cen, R. 1998, ApJ, 509, 494

Colberg, J. M., White, S. D. M., Yoshida, N., et al. 2000, MNRAS, 319, 209

Couchman, H. M. P. 1991, ApJ, 368, L23

de Theije, P. A. M., van Kampen, E., \& Slijkhuis, R. G. 1998, MNRAS, 297, 195

Diaferio, A., Kauffmann, G., Colberg, J. M., \& White, S. D. M. 1999, MNRAS, 307, 537

Einasto, M., Einasto, J., Heinämäki, P., et al. 2002, A\&A, submitted

Eke, V. R., Cole, S., \& Frenk, C. S. 1996, MNRAS, 282, 263

Fadda, D., Girardi, M., Giuricin, G., Mardirossian, F., \& Mezzetti, M. 1996, ApJ, 473, 670

Feller, W. 1966, An Introduction to Probability Theory and its Applications, vol. II (John Wiley \& Sons, Inc., New York)

Garcia, A. M. 1993, A\&AS, 100, 47

Girardi, M., Borgani, S., Giuricin, G., Mardirossian, F., \& Mezzetti, M. 1998, ApJ, 506, 45

Girardi, M., \& Giuricin, G. 2000, ApJ, 540, 45

Governato, F., Babul, A., Quinn, T., et al. 1999, MNRAS, 307, 949

Hanany, S., Ade, P., Balbi, A., et al. 2000, ApJ, 545, L5

Jenkins, A., Frenk, C. S., Pearce, F. R., et al. 1998, ApJ, 499, 20

Jenkins, A., Frenk, C. S., White, S.D.M., et al. 2001, MNRAS, 321, 372

Kauffmann, G., Colberg, J. M., Diaferio, A., \& White, S. D. M. 1999, MNRAS, 303, 188

Knebe, A., Kravtsov, A. V., Gottlöber, S., \& Klypin, A. A. 2000, MNRAS, 317, 630

Lahav, O., Bridle, S. L., Percival, W. J., et al. 2002, MNRAS, 333, 961

Lin, H., Kirshner, R. P., Shectman, S. A, et al. 1996, ApJ, 464, 60

Martínez, H. J., Zandivarez, A., Merchán, M. E., \& Domínguez, M. J. L. 2002 [astro-ph/0208425]

Moore, B., Frenk, C. S., \& White, S. D. M. 1993, MNRAS, 261, 827

Peebles, P. J. E. 1993, Principles of Physical Cosmology (Princeton University Press, Princeton, NJ)

Pierpaoli, E., Scott, D., \& White, M. 2001, MNRAS, 325, 77

Power, C., Navarro, J. F., Jenkins, A., et al. 2002, MNRAS, submitted [astro-ph/0201544]

del Popolo, A., \& Gambera, M. 2000, A\&A, 357, 809325

del Popolo, A., Gambera, M., Recami, E., \& Spedicato, E. 2000, A\&A, 353, 427

Press, W. H., \& Schechter, P. 1974, ApJ, 187, 425

Ramella, M., Pisani, A., \& Geller, M. J. 1997, AJ, 113, 483

Reiprich, T. H., \& Böhringer, H. 2002, ApJ, 567, 716

Seljak, U., \& Zaldarriaga, M. 1996, ApJ, 469, 437

Shectman, S., Landy, S., Oemler, A., et al. 1996, ApJ, 470, 172

Splinter, R. J., Melott, A. L., Shandarin, S. F., \& Suto, Y. 1998, ApJ, 497, 38

Suisalu, I., \& Saar, E. [astro-ph/9511120]

Tucker, D. L., Oemler, A. Jr., Hashimoto, Y., et al. 2000, ApJS, 130, 237 (TUC)

Viana, P., \& Liddle, A. R. 1996, MNRAS, 281, 323

White, S. D. M., Efstathiou, G., \& Frenk, C. S. 1993, MNRAS, 262, 1023 